Case report

\title{
Cardiac tamponade associated with a peripheral vein central venous catheter
}

\author{
MARÍA J. COLOMINA MD*, CARMEN GODET MD*, \\ FERRAN PELLISÉ MD†, MIGUEL ÁNGEL GONZÁLEZ-P \\ $\mathrm{MD}^{*}$, JOAN BAGÓ MD† AND CARLOS VILLANUEVA MD† \\ *Department of Anaesthesiology, +Department of Orthopedic Surgery, Hospital Universitario \\ Vall d'Hebron, Área de Traumatología Passeig Vall d'Hebron, Barcelona, Spain
}

\begin{abstract}
Summary
We present a case of cardiac tamponade associated with placement of a central venous catheter $(\mathrm{CVC})$ via a peripheral vein in a 14-year-old girl with idiopathic scoliosis undergoing corrective surgery. A number of complications have been described in association with CVC misplacement. Sporadic cases of cardiac tamponade from this have been reported, but the actual incidence is unknown. Death from cardiac tamponade attributed to CVCs ranges from 65 to 100\%. In our patient, cannulation of the pericardiophrenic vein was probably the cause of cardiac tamponade, based on radiological evidence that the initial location of the catheter was near the right atrium and possibly at the outlet of the pericardiophrenic vein. The catheter could have advanced into the vein and then to the pericardial sac with postural changes. The acute clinical course of cardiac tamponade in our patient had potentially lethal hemodynamic repercussions. The main diagnostic test for this condition is echocardiography and the only effective treatment is drainage of the pericardial effusion. Echocardiography should be performed before pericardiocentesis except in life-threatening situations or high clinical suspicion. Although they are rare, it is important to be aware of the potential for CVC complications.
\end{abstract}

Keywords: cardiac tamponade, central venous catheter complications, pericardiophrenic vein, scoliosis surgery

The iatrogenic causes of cardiac tamponade (CT) include, by frequency, cardiac surgery, coronary artery catheterization and central venous catheter (CVC) placement (1). Malposition of a CVC has an overall incidence of $1-6 \%$ (2). Because of the severity of this clinical condition, it is essential to recognize the signs of CT, which if not diagnosed and treated, has a fatal outcome in the majority of cases (2-5).

Correspondence to: Maria J. Colomina, C/Rosselló, 72, 3º-1, 08029, Barcelona, Spain (email: 26750mjc@comb.es/mjcolomina@ vhebron.net).
We present a case of CT associated with placement of a CVC via a peripheral vein in a patient with idiopathic scoliosis undergoing corrective surgery.

\section{Case report}

A 14-year-old girl (weight $40 \mathrm{~kg}$ ) with no associated pathology and classified as ASA I was scheduled for corrective surgery for idiopathic scoliosis (angular magnitude $87^{\circ}$ from $\mathrm{T} 4$ to $\mathrm{T} 11$ and $47^{\circ}$ from T12 to L4). In the preoperative evaluation moderate

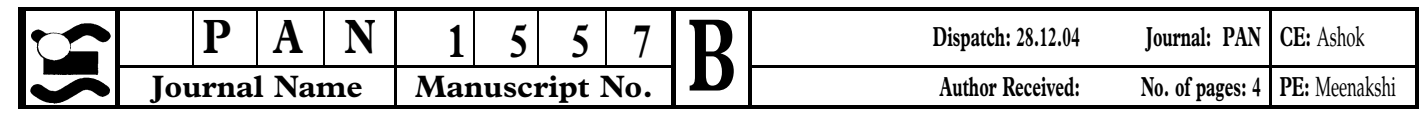


restrictive changes were seen on respiratory function testing. Doppler echocardiography, ECG, chest $\mathrm{X}$-ray and laboratory analyses were normal.

After induction of anesthesia, invasive hemodynamic monitoring was started. A 14-G Drum ${ }^{\circledR}$ polyurethane CVC (Drum-Cartridge Catheter, Abbott, Ireland) was inserted by peripheral access (left basilic vein) for perfusion of crystalloid solutions, and a cannula was placed in the left radial artery for continuous recording of pressures (monitor, DatexOhmeda S/5 Instrumentarium Corp., Finland).

As confirmed by a plain chest X-ray, the CVC tip was situated at the limit of what seemed to be the right atrium (Figure 1). The patient was positioned prone on a Relton Hall operating frame and the surgical procedure started. This consisted of spinal fusion from T2 to L3 plus right costoplasty, which lasted approximately $360 \mathrm{~min}$.

There were no incidents related to hemodynamic, respiratory or spinal function during surgery. Approximately $3 \mathrm{~h}$ after insertion of the CVC, the monitor showed elevated central venous pressure $(+15 \mathrm{mmHg})$ and the pressure trace was not typical of the superior vena cava-right atrium. It was decided to stop the crystalloid infusion (total given $350 \mathrm{ml}$ ) and withdraw the catheter.

Coinciding with the end of the procedure, there was gradual, progressive arterial hypotension and supraventricular tachycardia that showed transient

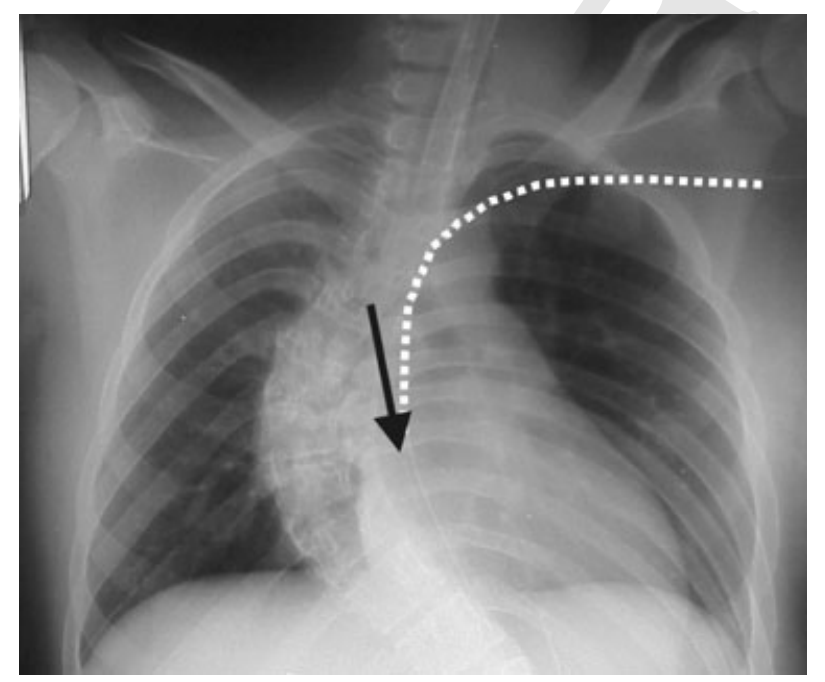

Figure 1

Radiological confirmation after CVC placement $(\rightarrow$ approximate location of the catheter tip). improvement with administration of intravenous crystalloids and colloids. Acute anemia or insufficient analgesia and sedative hypnotic drugs were ruled out, as was a possible acute respiratory problem, such as pleural perforation from surgical trauma. When the patient was positioned supine, clinical evidence of jugular venous distension, cyanosis, hypotension, tachycardia, abdominal distension, and decreased heart sounds were observed. On the basis of suspected obstructive cardiogenic shock, vigorous treatment was initiated with fluid volume, adjustment of ventilatory support and administration of vasoactive, inotropic drugs.

Subsequently, the patient was transferred to the recovery room with mechanical ventilation. Analytic parameters (arterial blood gas, $\mathrm{PaO}_{2} / \mathrm{FiO}_{2}$ ) and chest $\mathrm{X}$-ray findings (Figure 2) showed progressive normalization, and at $24 \mathrm{~h}$ mechanical ventilation was withdrawn.

On echocardiography at $24 \mathrm{~h}$ and on the fifth postoperative day, there were no changes in the cardiac chambers or pericardial sac. The postoperative course on the ward was uneventful and the patient was discharged from the hospital 9 days after surgery.

At later follow-up visits the patient remained asymptomatic and there were no sequelae attributable to CT.

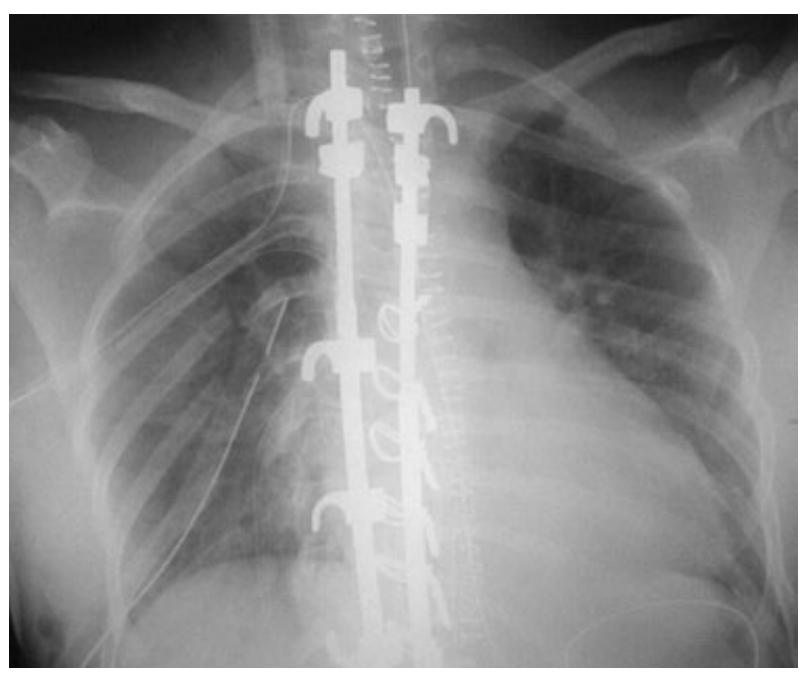

Figure 2

X-ray immediately after pericardiocentesis. 


\section{Discussion}

A number of complications have been described in association with CVC misplacement, including arterial puncture, pneumothorax, hemothorax, cardiac dysrhythmia, Horner's syndrome, brachial plexus lesion, laceration of a vertebral artery, thoracic duct lesion, air embolism and tracheal laceration (4). Several risk factors inherent to CVC insertion have been identified (2-6) such as the venous route selected, the position and movement of the catheter tip with postural changes, the angle of the tip against the vessel or cardiac walls and the material used for catheters and insertion guidewires. The potential danger of incorrect catheter tip position seems to be particularly related to its movement during changes in arm, neck or head position and after changing to the decubitus. Abduction and elevation of the arm in a patient with a CVC inserted thorough an antecubital vein can cause the catheter tip to advance as much as $7 \mathrm{~cm}$ (5). Cardiac tamponade is more frequently related to CVCs inserted through a peripheral vein than those placed directly in a central vein (5).

The review by Mhun et al. (6) of 2580 patients with a CVC showed catheter tip malposition in $1.1 \%$ for the right internal jugular vein and $4 \cdot 2 \%$ for the left. Among these patients, cannulation of small veins was documented in only two cases.

Cardiac tamponade, which was probably caused by cannulation of a small vein (pericardiophrenic) in our patient, is an extremely rare complication $(4,6)$. Sporadic cases of CT caused by CVC placement have been reported in adults, children and neonates $(3,7)$, but the actual incidence is unknown (7). Death from CT attributed to CVCs ranges from 65 to $100 \%$ (5).

There are few references on pericardiophrenic vein cannulation $(6,8-10)$, since it is difficult to make the diagnosis. This vein drains into one or other side of the brachiocephalic vein, in front of the internal jugular vein (Figure 3). Our conclusion that cannulation of this vein was the cause of CT is based on radiological evidence that the initial location of the catheter was near the right atrium and possibly at the outlet of the pericardiophrenic vein; with postural changes the catheter could have advanced into the vein and then to the pericardial sac (Figures 1 and 3). The clinical changes and the fact that the pericardiocentesis fluid was not bloody, together

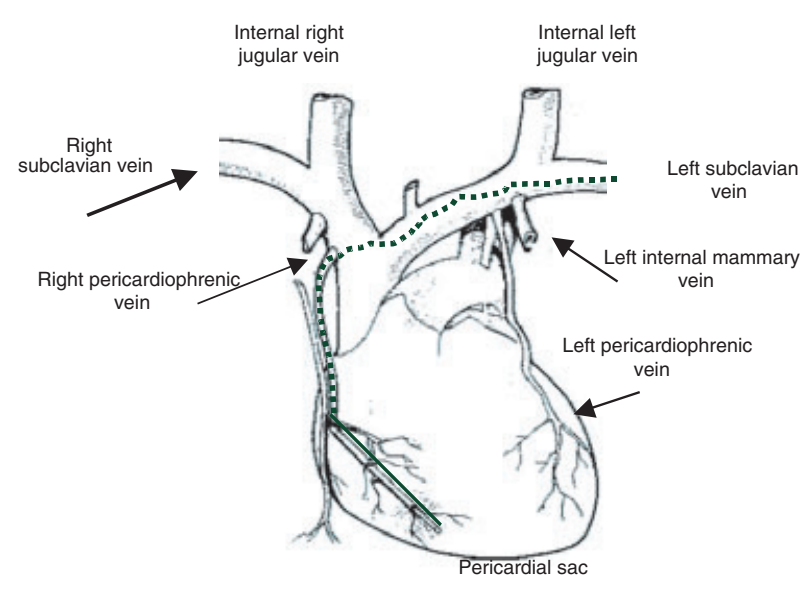

Figure 3

Probable course of the CVC from the left internal subclavian vein to the right pericardiophrenic vein and the interior of the pericardial sac.

with the later recovery without abnormal findings on echocardiographic follow-up, also support this conclusion.

Although chest radiography cannot rule out the development of complications from catheter tip malposition (4), it should always be performed at the time of insertion to confirm the position $(3,5)$. Several guidelines have been formulated for proper CVC placement with radiological monitoring, however, none can guarantee an absence of risk. The classic criterion of Greenall (5) suggests that the catheter tip should lie less than $2 \mathrm{~cm}$ below the line that joins the lower border of the medial ends of both clavicles when the patient is in a standing position (5). However, such references are not valid for a supine patient on the operating table. In an attempt to resolve this, another anatomical landmark at the junction of the right main bronchus and the trachea, which represents the upper limit of the pericardial reflection over the lowest portion of the superior vena cava has been suggested (4). Some authors (3, 11) place the landmark at the third costosternal junction, the T5 level (T2 for neonates) (3), or at $2 \mathrm{~cm}$ from the pericardial reflection (11). Intracardiac localization of a CVC is questionable because of the potential risk involved $(3,11)$.

Cardiac tamponade has potentially lethal hemodynamic repercussions from accumulation of fluid in the pericardial space, which triggers collapse of the heart chambers and a fall in cardiac output (12). The most important factors involved are the 
relationship between pericardial pressure and volume, and the speed with which fluid accumulates. Compensating mechanisms mediated by increased adrenergic tone are activated in response to CT. These mechanisms remain active for a limited period and, depending on the intensity and speed of the hemodynamic changes caused by the pericardial effusion $(12,13)$. The pericardial pressure/volume relationship explains why our patient showed such rapid improvement after only a small part of the pericardial effusion was aspirated.

The clinical picture of acute CT involves hypotension, increased central venous pressure and circulatory collapse. This condition should be suspected when a patient develops shock with jugular venous distension, paradoxical pulse and tachycardia which can go on to bradycardia and cardiac arrest. Mechanical ventilation during CT should be tailored to the patient's needs, since an increase in intrathoracic pressure can worsen the clinical process $(3,5,12,13)$.

The main diagnostic test in CT is echocardiography $(2-5,7,12,13)$, which detects the pericardial effusion and determines cardiac dynamics. The differential diagnosis is between cardiac or pulmonary etiologies. Among the cardiac causes, constrictive pericarditis, restrictive cardiomyopathy, and right ventricular infarction should be considered. Among the pulmonary causes, pulmonary thromboembolism, tension pneumothorax and cor pulmonale must be excluded. The mechanical ventilation parameters in our patient showed no airway pressure or endtidal $\mathrm{CO}_{2}$ changes and there were no ST-segment changes in the ECG.

The only effective treatment for this condition is drainage of the effusion $(2-5,7,12,13)$. Echocardiography should be performed before pericardiocentesis except in life-threatening situations or high clinical suspicion (13). Our patient was able to undergo echocardiography because her hemodynamic status improved initially with intravenous fluid administration and pharmacological support and the echocardiograph was immediately available in the operating room. Supportive treatment is based on increasing the preload and on inotropic and vasoactive agents. Diuretics and vasodilators are totally contraindicated $(12,13)$.

In summary, CVC placement should be used only when indicated and should be removed immedi- ately after fulfilling its purpose. The site of the catheter tip can change with respiratory or cardiac movements or postural changes. It should be confirmed that the catheter tip is not in an intracardiac position. One useful reference position is the third costosternal junction or the T5 level (T2 for neonates). Radiographic confirmation of this position is recommended, particularly in pediatric patients (3). The diagnosis of CT is based on clinical criteria and it may or may not be confirmed with additional investigations. Sometimes these studies cannot be performed because of the urgency of the situation. It is important to be aware of the potential for CVC complications, although they are rare. Confirmation of a proper initial position does not preclude the development of a complication, including migration of the device.

\section{References}

1 Forauer AR, Dasika NL, Gemmete J et al. Pericardial tamponade complicating central venous interventions. J Vasc Interv Radiol 2003; 14: 255-259.

2 Yavascaoglu B, Yilmazlar A, Korfali G. Pericardial tamponade as a delayed lethal complication of central venous catheterization. Eur J Anaesth 2001; 18: 485-489.

3 Nadroo AM, Lin J, Green RS et al. Death as a complication of peripherally inserted central catheters in neonates. I Pediat 2001; 138: 599-601.

4 Losert H, Prokesch R, Grabenwöger $M$ et al. Inadvertent transpericardial insertion of a central venous line with cardiac tamponade failure of preventive practices. Intens Care Med 2000; 26: 1147-1150.

5 Booth SA, Norton B, Mulvey DA. Central venous catheterization and fatal cardiac tamponade. Br J Anaesth 2001; 87: 298302.

6 Khan F, Khan F. A rare misplacement of a central venous catheter. Eur J Anaesth 2000; 17: 527-528.

7 Bagwell CE, Salzberg AM, Sonnino RE et al. Potentially lethal complications of central venous catheter placement. J Pediatr Surg 2000; 35: 709-713.

8 Ruschulte H, Panning B, Jaeger K. Control of central venous catheter position. Eur J Anaesthes 2001; 18: 485-489.

9 Luks F, St-Vil D, Angaté H et al. Inadvertent pericardiacophrenic vein catheterization. Chest 1992; 102: 1635.

10 Zamam MH, Mitra P, Bondi E et al. A rare malposition of the central venous catheter. Chest 1990; 98: 768-770.

11 Collier PE, Goodman GB. Cardiac tamponade caused by central venous catheter perforation of the heart: a preventable complication. J Am Coll Surg 1995; 181: 459-463.

12 Shoemaker WC. Textbook of Critical Care, 4th edn.

1 Panamericana: xxxx, 2000: 1076-1079.

13 Schaller MD, Eckert P, Tagan D. Choc cardiogénique. Encycl Méd Chir. París: Elsevier, Anesthésie-Réanimation, 36-840-c-10, 1999: 14.

Accepted 28 October 2004 